\title{
Inverse Multifractal Analysis of Different Frame Types of Multiview 3D Video
}

\author{
Amela Zeković and Irini Reljin
}

\begin{abstract}
In this paper, the results of multifractal characterization of multiview 3D video are presented. Analyses are performed for different views of multiview video and for different frame types of video. Multifractal analysis is performed by the histogram method. Due to the advantages of the selected method for determining the spectrum, the inverse multifractal analysis of multiview 3D video was also possible. A discussion of the results obtained by the inverse multifractal analysis of multiview $3 D$ video is presented, taking into account the frame type and whether the original frames belong to the left or right view of multiview 3D video. In the analysis, publicly available multiview 3D video traces were used.
\end{abstract}

Keywords - 3D video, frame types, inverse multifractal analysis, multiview coding.

\section{INTRODUCTION}

$\mathrm{T}$ HREE-DIMENSIONAL (3D) video is a video that creates an impression of depth for viewers [1]-[3]. This type of video usually contains several video sequences casing it to have an even larger quantity of data than the conventional single view video. Since a $3 \mathrm{D}$ video represents a large portion of data traffic in terrestrial and wireless networks, a thorough understanding of $3 \mathrm{D}$ video characteristics is required. Some previous research is devoted to encoding of 3D video [3], [4], while other studies concentrate on the transfer, storage and display of this type of video [5].

It was shown that, due to variability, compressed video sequences such as MPEG-4, H.263, and H.264, have fractal properties, [6], [7]. We have analyzed 3D video from a multifractal point of view and computed the multifractal spectra by the histogram method. From the multifractal spectra derived by the histogram method, it is possible to perform inverse multifractal analysis. We

Paper received March 19, 2014; revised September 23, 2014; accepted September 25, 2014. Date of publication November 15, 2014. The associate editor coordinating the review of this manuscript and approving it for publication was Prof. Ljiljana Milić.

This paper is a revised and expanded version of the paper presented at the 21th Telecommunications Forum TELFOR 2013.

This research is partially funded by the Serbian Ministry of Education, Science and Technology Development under grant No.III44009.

Corresponding author Amela Zeković is with the University of Belgrade, School of Electrical Engineering, Bulevar kralja Aleksandra 73, 11120 Belgrade, Serbia and with School of Electrical and Computer Engineering of Applied Studies, Vojvode Stepe 283, Belgrade, Serbia (e-mail: amelaz@viser.edu.rs).

Irini Reljin is with the University of Belgrade, School of Electrical Engineering, Bulevar kralja Aleksandra 73, 11120 Belgrade, Serbia (e-mail: irinitms@gmail.com). performed inverse multifractal analysis of 3D video, while paying attention to different frame types in the video and different views of the video. Our analysis is focused on a multiview 3D video format, which is a standard of video coding developed by MPEG/VCEG that is included as an amendment to H.264/MPEG-4 AVC video compression standard [8]. Analyses were conducted using publicly available frame size video traces [9], [10].

The paper is organized as follows: Section II gives a review of multiview $3 \mathrm{D}$ video traces, while Section III is dedicated to the basics of multifractal and inverse multifractal analysis. Simulation results are presented in Section IV. Some concluding remarks are given in Section V.

\section{Multiview 3D VideO Traces}

Multiview video contains several video sequences representing different views of the same scene [1]-[5]. Combining these video sequences, an observer has an impression of depth, so this video is referred to as a $3 \mathrm{D}$ video. The amount of information in a multiview $3 \mathrm{D}$ video is significantly higher than in a classic video that contains one video sequence, since every view in multiview $3 \mathrm{D}$ video content has one video sequence - a series of frames [1], [2], [5]. If each view is encoded using some of the classic codecs (such as H.264/AVC), the amount of information becomes larger as many times as there are views over the video with one view, but it is simple to decode a legacy 2D (two-dimensional) video format. Since the amount of information is very important for the video transmission and storage, it is necessary to reduce it as much as possible. Coding efficiency of multiview 3D videos can be enhanced using inter-view redundancy, which means that in addition to time reference images, this type of coding uses inter-view reference images, according to [8]. This approach to coding is added as an amendment to the H.264/AVC standard to support multiview coding [8]. Video content that contains opposite views of the scene is referred to as a stereoscopic video. For 3D video with two views, this means that when coding a view taken as a reference (usually the left), the second view is coded relative to the reference, resulting in a reduction in the amount of information. In this setup, the left view has I, P and $\mathrm{B}$ frames (intra-coded, predictive, bidirectional), while the right view contains only $\mathrm{P}$ and $\mathrm{B}$ frames [5].

In this paper, the analysis of multiview $3 \mathrm{D}$ video sequences by using video traces is performed, using traces publicly available at [10]. The procedure of obtaining the video traces, and details about them, can be found in [5], [9]. For the 3D video, movie "Alice in Wonderland" 
directed by Tim Burton (2010) is selected. Analysis is performed on traces of multiview video that contains two views (left and right), where each view has 51184 frames. These video traces contain data about the frame number, frame time, its type, size and peak signal-to-noise ratio. In the analysis, we used data about the number, type, and size of the frame. Organization of GoP (Group of Pictures), for the analyzed video is G16B1, which means that the left view has the frame organization IВРВРВРВРВРВРВРВІ..., while the structure of the pictures for the right view is РВРВРВРВРВРВРВРВР... In the analysis, we observed video content with quantization parameters (I, P, B) $(28,28,28)$. The analysis is performed for the combined view, where the view is created using the frames of the left and right view. Video content is in full HD (High Definition) resolution, with $1920 \times 1080$ pixels, and with the frame rate of 24 frames/s for each view. Coding of the signal is performed using JMVC 8.3.1 coder.

\section{Multifractal AND InVERSE MultifRACTAL ANALYSIS}

Fractals are complex geometric objects with fine structure at an arbitrary small scale. These objects can have a property of self-similarity, which means that regardless of how complex the shape and/or dynamic behavior of the system is found at one scale, resembling objects could be found in other scales [11], [12].

Natural objects and phenomena can have self-similar properties exposing statistically. For example, some natural objects, like mountains, clouds, coastlines, blood vessel networks, expose self-similar properties in a manner that in different scales the shape of the object is not exactly the same, but similar. Objects that possess this property are labeled as multifractals. Modern communication traffic, some geographic and meteorological phenomena, fluid mechanics, biomedical signals, statistic data about population, expose the multifractal property.

The properties of fractal signals and objects are [12]: fractal structures do not have length that describes them, property of self-similarity, and fractional (non-integer) dimension. The main analytical parameter for describing structures that have scaling symmetry is the fractal dimension. The term scalable symmetry implies selfsimilarity of the observed objects in variable scale. The first introduction of measures to fill the area that allows the possibility of fractional dimension was given by the German mathematician Hausdorff. Besides the Hausdorff definition explained in [11], the fractal dimension can be calculated in other ways. Furthermore, for the structures that are obtained by applying strictly defined rules, the dimension self-similarity can be determined [11]. However, for fractal structures that are not acquired using strictly defined rules, fractal dimension cannot be defined as a dimension of self-similarity. In that case, other methods might be used, among which the best known is the box-counting method, or the method of overlays, being used to determine the dimension of overlay.

For a complete characterization of multifractals, dimension as a single number is not enough, so some kind of distribution function is necessary. Thus, for the characterization of multifractals a value named coarse Hölder exponent, labeled by $\alpha$, is introduced as

$$
\alpha=\frac{\log \mu(\text { box })}{\log \varepsilon},
$$

where $\mu$ is the measure of the boxes that are used to cover the multifractal structure, and $\varepsilon$ is the dimension of the box. For a wide class of self-similar measures, $\alpha$ takes a value within the interval $\left[\alpha_{\min }, \alpha_{\max }\right]$, where $0<\alpha_{\min }<\alpha_{\max }<\infty$. Values of $\alpha$ are close to the corresponding fractal dimension of the observed structures.

Within the observed structure many points can be characterized by the same value of $\alpha$. It is possible to observe the distribution of the exponent $\alpha$ as another parameter to characterize the multifractal structures. For each value of $\alpha$ we can count boxes sized $\varepsilon$ having the coarse Hölder exponent equal to $\alpha$. If this number, denoted as $N_{\varepsilon}(\alpha)$, is determined, the Hausdorff dimension of the distribution of $\alpha$ is defined as

$$
f_{\varepsilon}(\alpha)=-\frac{\log N_{\varepsilon}(\alpha)}{\log \varepsilon} .
$$

When $\varepsilon \rightarrow 0$ the function given by (2) approaches a limiting value $f(\alpha)$ which is known as multifractal spectrum. Note that direct determination of (2) is not possible since the logarithm of zero is infinite. This value can be estimated from the slope of the log-log plot for several values of $\varepsilon$. The value of $f(\alpha)$ could be interpreted as a fractal dimension of subsets of boxes of size $\varepsilon$ with the coarse Hölder exponent equal to $\alpha$.

There are several algorithms for the calculation of $f(\alpha)$ . For instance, the method based on statistical moments, then on the basis of geometric parameters and probability theory [11]. According to the classification given in [11], there are three types of spectra of $f(\alpha)$ : the Hausdorff singularity spectrum, $f_{h}(\alpha)$, Large deviations spectrum, and the Legendre multifractal spectrum, $f_{l}(\alpha)$.

For obtaining an estimate of $f(\alpha)$ for our data, we used the histogram method [11], [13], [14]. This method for a given measure $\mu$ involves the following steps:

1) covering the measure with boxes of size $\varepsilon$ and determining the number of boxes $N_{\varepsilon}(\alpha)$,

2) compute coarse Hölder exponent $\alpha_{i}$, where $\mu_{i}$ is a measure of box $i$,

3) making histogram for $\alpha$,

4) repeat step 3 for different values of $\varepsilon$,

5) plot $-\log N_{\varepsilon}(\alpha) / \log \varepsilon$ versus $\alpha$ for different values of $\varepsilon$,

6) estimate the $f(\alpha)$ from the slope of plots in 5) for several values of $\varepsilon$.

Based on the procedure for determining a multifractal spectrum by the histogram method, it is possible to carry 
out the procedure of inverse multifractal analysis (IMF), as well. This procedure is suggested in [13] and derived in [14] as a computer routine HISTMF. The extension of HISTMF is applied to the detection of microcalcifications in digital mammograms [15] and embedded as the module for extracting small details in images in the computer package FracLab [16]. The inverse multifractal analysis means that from one derived MF spectrum it is possible to recognize and extract signal parts having particular values of the pair $(\alpha, f(\alpha))$. This procedure is particularly interesting in regions where the MF spectrum takes a small value (globally rare cases), and in regions where the value of $f(\alpha)$ is large (globally frequent cases). Another interesting point is to create and extract expressed local fractal behavior (large values of $\alpha$ ), that is an indication of smooth signal and small changes and weak local fractal behavior (small values of $\alpha$ ), that is an indication of burstier signal.

\section{Simulation Results}

Multifractal analysis is applied to multiview 3D video trace Alice in Wonderland for the frame size data (video traces). A detailed description of used video traces is given in Section II. The multifractal spectra of Hausdorff singularity were obtained by the histogram method [14] using the code particularly developed for this purpose. The results for the multifractal spectrum for intra-coded (I), predictive $(\mathrm{P})$, and bidirectional (B) frames of the combined view of the multiview $3 \mathrm{D}$ video are presented in Fig. 1.

Our previous analysis given in [17] contains results for multifractal and inverse multifractal tests for all frames of the left and right views of the multiview 3D video, regardless of their type.

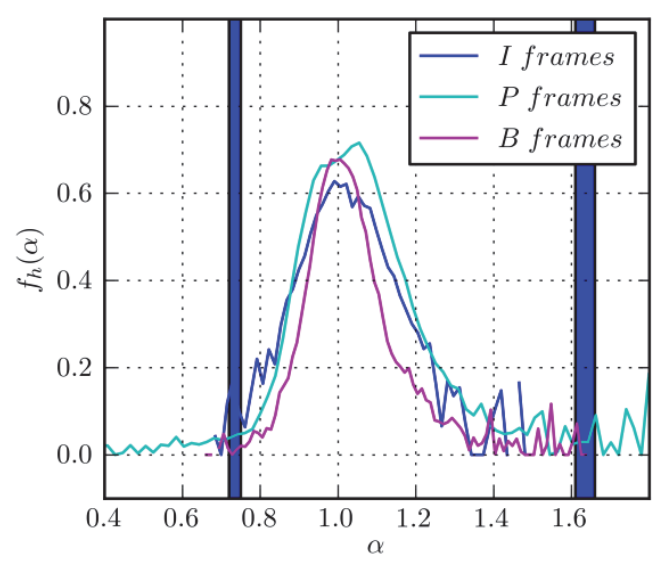

Fig. 1. Multifractal spectra by the histogram method for I, P, and B frames of the combined view for multiview $3 \mathrm{D}$ video.

Analyzing Fig. 1, where the multifractal spectra of the selected multiview 3D signal for different frame types are depicted, depressions in the $f_{h}(\alpha)$ spectrum for $\alpha$ values close to 0.8 , and close to 1.6 can be observed (highlighted bands in Fig. 1). The same bands of $\alpha$ are observed in our analysis given in [17]. In the case of these local singularities, the value for $f(\alpha)$ is very small, which means that these are rare events. Inverse multifractal analysis was conducted for these bands of $\alpha$ and $f(\alpha)$ values and for different frame types.

Fig. 2 (a) provides an overview of frame sizes of I frames of the selected video trace, while Fig. 2 (b) shows isolated those frame sizes of I frames that have $\alpha$ in the range $0.72<\alpha<0.75$. For I frames there are no frame sizes with $\alpha$ in the range $1.61<\alpha<1.66$, because, as can be seen in Fig. 1, multifractal spectrum for I frames has $\alpha_{\max }$ lower than the values in the examined range. Extracted frames that are shown in Fig. 2 (b) are rare events, when a major change occurs in the video content. Frames separated in Fig. 2 (b), are some of the largest frames in the video trace.

Fig. 3 and Fig. 4 show the results of the inverse multifractal analysis for $\mathrm{P}$ and $\mathrm{B}$ frames, respectively. Analysis is performed for the small values of $\alpha$ ( $0.72<\alpha<0.75)$, and for the high values of $\alpha$ ( $1.61<\alpha<1.66)$. The results of inverse multifractal analysis for the lower range of $\alpha$ values show some of the largest $\mathrm{P}$ frames in the video trace in Fig. 3 (b) and some of the largest B frames in Fig. 4 (b). The results of inverse multifractal analysis for the higher range of $\alpha$ values show the opposite, the smallest $\mathrm{P}$ and $\mathrm{B}$ frames in Fig. 3 (c) and in Fig. 4 (c), respectively. The difference in the number of frames shown in Fig. 3 and Fig. 4 is the result of $f(\alpha)$ parameter values where a greater $f(\alpha)$ leads to a larger number of frames.

The results of the inverse multifractal analysis for different frame types of the combined view of multiview $3 \mathrm{D}$ video are analyzed further. The combined view of the analyzed video consists of the left and right views, as explained in Section II. Frames of the left and right view appear alternately in the combined view. This feature is utilized to determine the origin of frames obtained in the inverse multifractal analysis of the combined view. Results of the inverse multifractal analysis reveal that the frames of the right view are smaller in size than the frames of the left view. This is a consequence of the manner of coding of multiview 3D video, where the frames of the left view are used as a reference for coding the frames of the right view.

\section{CONCLUSION}

In this paper, the results of the multifractal characterization of multiview $3 \mathrm{D}$ video content are presented. For the analysis, a multiview $3 \mathrm{D}$ video compression format is selected, as $3 \mathrm{D}$ video coding that is included as an amendment to H.264/MPEG-4 AVC video compression standard as a support for multiview coding. For the analysis of multiview 3D compression format, long frame-size traces are used, publicly available [10].

In the case of the multiview 3D video, where every view is a video sequence in full HD resolution, the amount of data is larger in comparison to other compression formats. Since video content presents a large portion of data traffic, its properties, characterization and modeling are very important. 


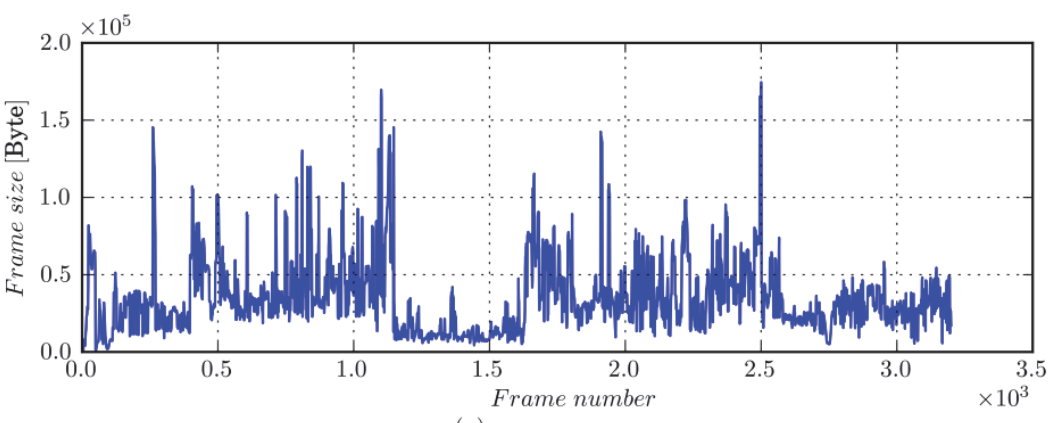

(a)

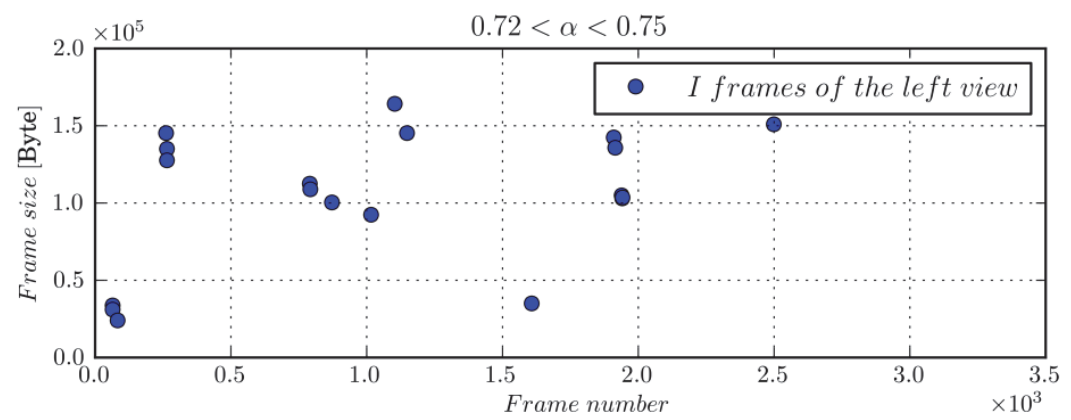

(b)

Fig. 2. Illustration of the inverse multifractal analysis of multiview 3D video Alice in Wonderland: (a) Frame sizes (video traces) of I frames for the combined view of multiview 3D video. (b) Extracted I frames that have $\alpha$ in the range $0.72<\alpha<0.75$.

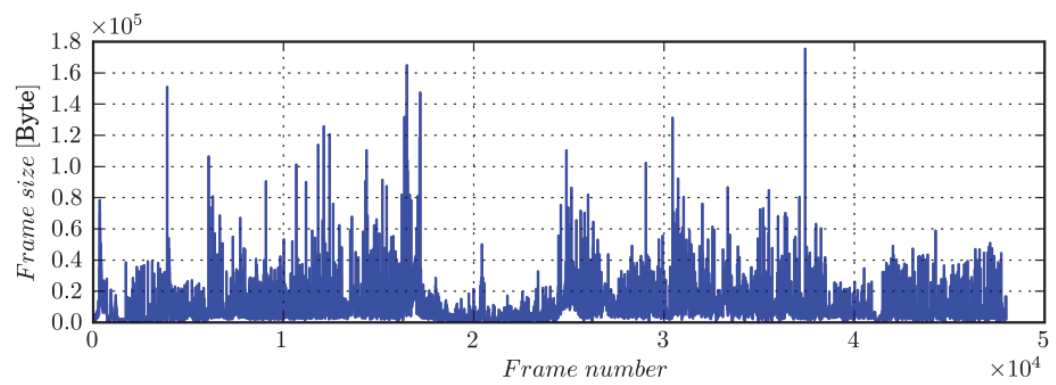

(a)

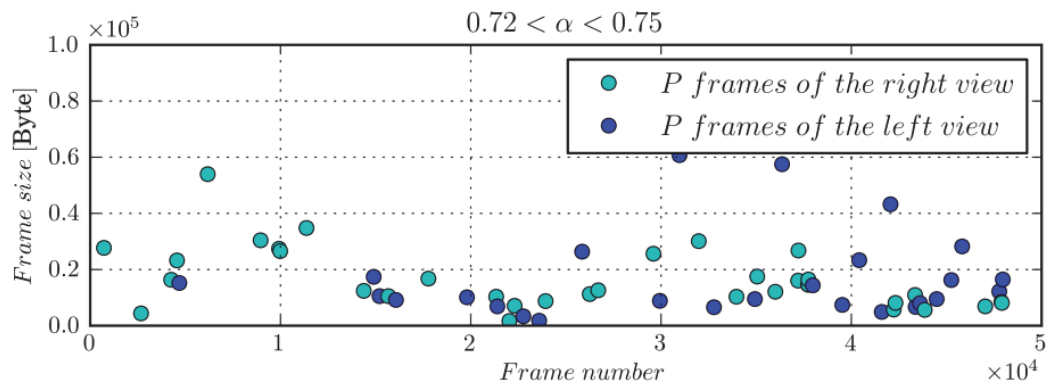

(b)

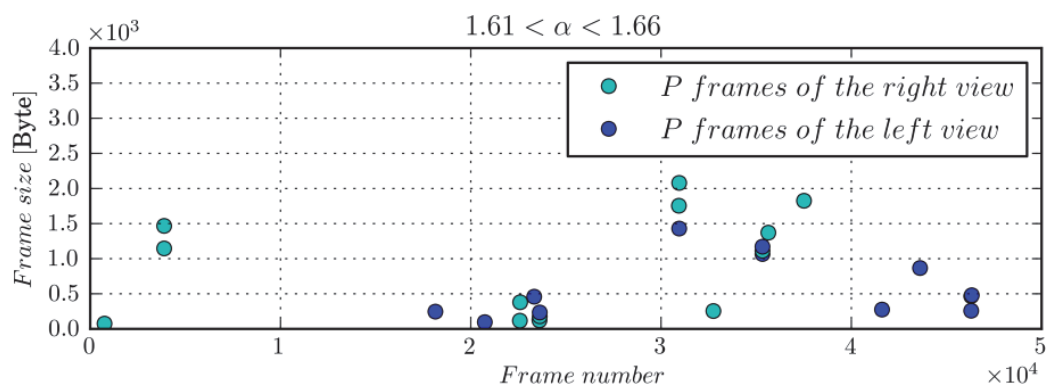

(c)

Fig. 3. Illustration of the inverse multifractal analysis of multiview 3D video Alice in Wonderland: (a) Frame sizes (video traces) of $\mathrm{P}$ frames of the combined view of multiview 3D video. (b) Extracted $\mathrm{P}$ frames for both views that have $\alpha$ in the range $0.72<\alpha<0.75$. (c) Extracted $\mathrm{P}$ frames for both views that have $\alpha$ in the range $1.61<\alpha<1.66$. 


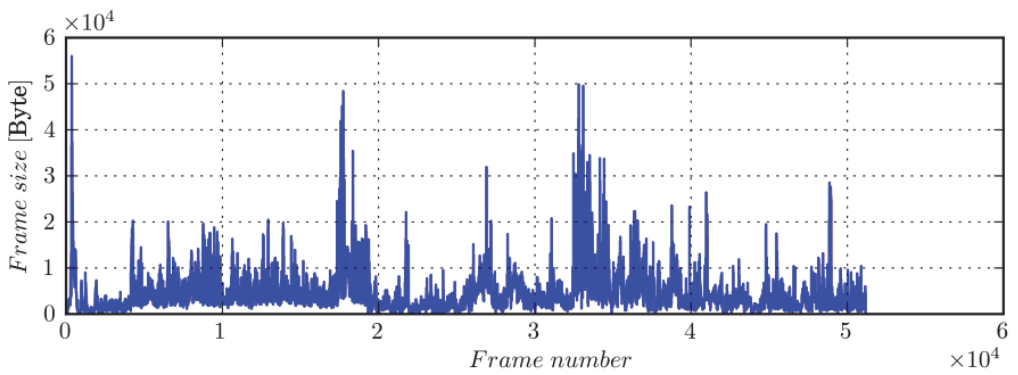

(a)

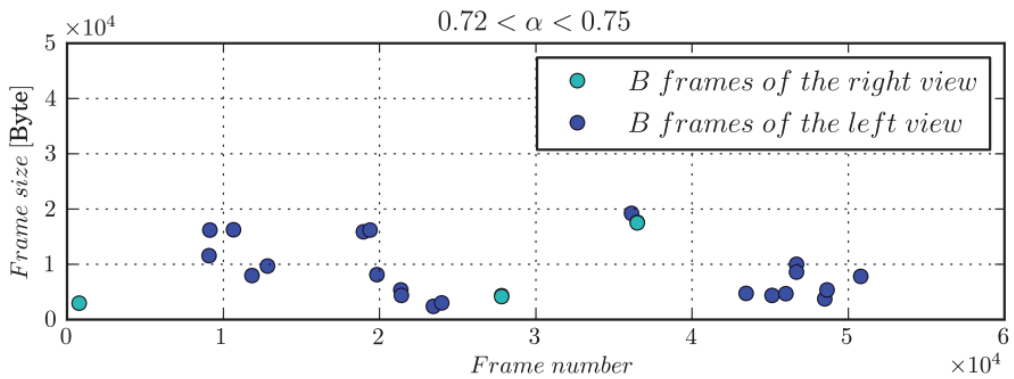

(b)

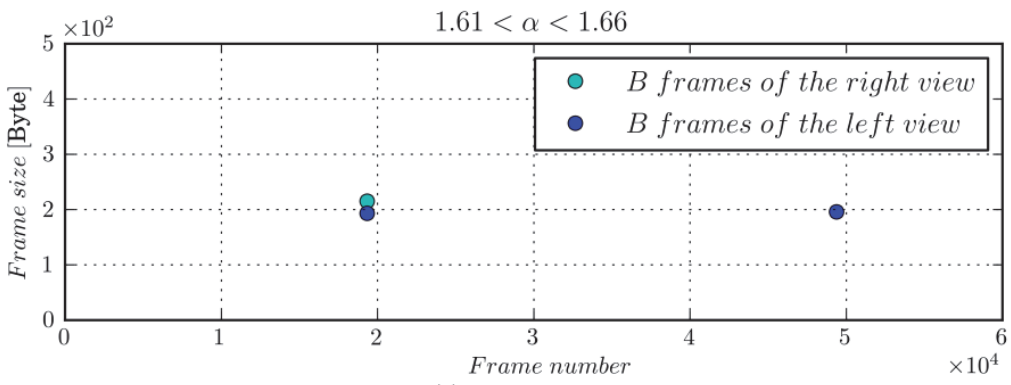

(c)

Fig. 4. Illustration of the inverse multifractal analysis of multiview 3D video Alice in Wonderland: (a) Frame sizes (video traces) of B frames of the combined view of multiview 3D video. (b) Extracted B frames for both views that have $\alpha$ in the range $0.72<\alpha<0.75$. (c) Extracted $\mathrm{B}$ frames for both views that have $\alpha$ in the range $1.61<\alpha<1.66$.

We have presented the results of detailed local and global fractal behavior of the multiview 3D video, for isolated I, P, and B frame types and for different views of the video. Also, the results of inverse multifractal analysis of the combined view are shown, successfully isolating some of the smallest and some of the highest I, P, and B frames in the video.

\section{REFERENCES}

[1] A. Smolic, K. Mueller, P. Merkle, C. Fehn, P. Kauff, P. Eisert, and T. Wiegand, "3D video and free viewpoint video-technologies, applications and MPEG standards," in Proc. IEEE International Conference on Multimedia and Expo, July 9-12, Toronto, 2006.

[2] P. Merkle, K. Muller, and T. Wiegand, "3D video: acquisition, coding, and display," IEEE Transactions on Consumer Electronics, vol. 56, no. 2, pp. 946-950, 2010.

[3] Y. Chen, Y.-K. Wang, K. Ugur, M. M. Hannuksela, J. Lainema, and M. Gabbouj, "The emerging MVC standard for 3D video services," EURASIP Journal on Advances in Signal Processing, 2009.

[4] S. Maiti, P. Desai, B. Patel, Y. Goel, E. M. Piccinelli, D. Aliprandi, P. Fragneto, and B. Rossi, "Smart 3D video coding," presented at the 3DTV-Conference: The True Vision - Capture, Transmission and Display of 3D Video (3DTV-CON), Zurich, Oct. 15-17, 2012.

[5] A. Pulipaka, P. Seeling, M. Reisslein, and L. Karam, "Traffic and statistical multiplexing characterization of 3D video representation formats," IEEE Transactions on Broadcasting, vol. 59, no. 2, pp. $382-389,2013$.

[6] I. Reljin and B. Reljin, "Fractal and multifractal analyses of compressed video sequences," Facta Universitatis Series: Electronics and Energetics, vol. 16, no. 3, pp. 401-414, 2003.
[7] I. Reljin, A. Samcovic, and B. Reljin, "H.264/AVC video compressed traces: Multifractal and fractal analysis," EURASIP Journal on Advances in Signal Processing, 2006.

[8] A. Vetro, T. Wiegand, and G. J. Sullivan, "Overview of the stereo and multiview video coding extensions of the H.264/MPEG-4 AVC standard," Proceedings of the IEEE, vol. 99, no. 4, pp. 626-642, 2011.

[9] P. Seeling, F. H. P. Fitzek, and M. Reisslein, Video Traces for Network Performance Evaluation. Dordrecht: Springer, 2007.

[10] Video trace library. Available: http://trace.eas.asu.edu

[11] A. Evertsz and B. Mandelbrot, "Multifractal measures," in Chaos and Fractals, H. Peitgen, H. Jurgens, and P. Andrews, Eds. New York: Springer, 1992, pp. 849-881.

[12] S. H. Strogatz, Nonlinear Dynamics and Chaos. Westview Press, 2001.

[13] J. L. Vehel, "Introduction to the multufractal analysis of images," Tech. Rep., INRIA, Rocquencourt, Le Chesnay Cedex, France, 1996.

[14] I. Reljin, B. Reljin, I. Pavlović, I. Rakočević, "Multifractal analysis of gray-scale images", in Proc. IEEE 10th Mediterranean Electrotechnical Conference, MELECON-2000, Vol. II, pp. 490493, Lemesos, Cyprus, May 29-31, 2000

[15] T. Stojic, I. Reljin, and B. Reljin, "Adaptation of multifractal analysis to segmentation of microcalcifications in digital mammograms," Physica A: Statistical Mechanics and its Applications, vol. 367, pp. 494-508, 2006.

[16] FracLab: A fractal analysis toolbox for signal and image processing, http://fraclab.saclay.inria.fr/

[17] A. Zekovic, I. Reljin, "Multifractal and inverse multifractal analysis of multiview 3D video," in Proc. of 21st Telecommunications Forum (TELFOR), Nov. 26-28, Belgrade, Serbia, 2013, pp. 753756. 